\title{
Some factors affecting separation and detection of amino acids by high-performance anion-exchange chromatography with integrated pulsed amperometric detection
}

\author{
Hong $\mathrm{Yu}^{\mathrm{a}, \mathrm{b}}$, Yong-Sheng Ding ${ }^{\mathrm{a}}$, Shi-Fen $\mathrm{Mou}^{\mathrm{a}, *}$ \\ ${ }^{a}$ Research Center for Eco-Environmental Sciences, Academia Sinica, P.O. Box 2871, Beijing 100085, China \\ ${ }^{\mathrm{b}}$ Department of Chemistry, Harbin Normal University, Harbin 150080, China
}

\begin{abstract}
Some factors influencing the separation and detection of amino acids by high-performance anion-exchange chromatography with integrated pulsed amperometric detection were investigated. These factors include eluent concentration, column temperature, and detection waveform. The selectivity changes in weakly retained amino acids are slight with changing sodium hydroxide eluent concentration. When sodium acetate eluent concentration is changed, the selectivity variations between strongly retained amino acids containing two carboxyl groups and containing only one carboxyl group are obviously different. Significant but slight selectivity changes in weakly retained amino acids can be achieved through changing the column temperature. Sodium hydroxide and sodium acetate eluent concentration affect the detection of amino acids. Detection sensitivity of amino acids can be improved by increasing the concentration of sodium hydroxide and sodium acetate in a certain concentration range. The detections of amino acids at two different detection waveforms were compared. The hydroxyl amino acids can be selectively detected by choosing a modified detection waveform. The optimized gradient elution condition and column temperature for analyzing 19 amino acids were obtained. The time for the gradient elution program was $60 \mathrm{~min}$. The column temperature was $35^{\circ} \mathrm{C}$. Under the optimized conditions, detection limits for 19 amino acids were $0.15-4.52$ pmol. The calibration graphs of peak area for all the analytes were linear for about three orders of magnitude. The RSDs $(n=5)$ of peak area were $0.6-5.6 \%$. The determination of trace amino acid impurities in valine product is shown as an application example.
\end{abstract}

(C) 2003 Elsevier Science B.V. All rights reserved.

Keywords: Mobile phase composition; Temperature effects; Detection, LC; Integrated pulsed amperometric detection; Electrochemical detection; Amino acids

\section{Introduction}

Amino acid analysis has an important role in the study of composition of proteins, foods and feedstuffs. Free amino acids are also determined in wine,

\footnotetext{
*Corresponding author. Tel.: +86-10-6284-9182; fax: +86-106284-9239.

E-mail address: shifenm@mail.rcees.ac.cn (S.-F. Mou).
}

fruit juice and biological material. At the present time, separations and determinations of amino acids are carried out mainly by cation-exchange chromatography with post-column derivatization and spectrophotometric detection [1-4], reversed-phase highperformance liquid chromatography with pre-column derivatization and spectrophotometric detection [58], gas chromatography with derivatization [9] and capillary electrophoresis $[10,11]$. In the above meth- 
ods, derivatization is usually employed. The determination is thus complicated. In 1999, Clarke et al. [12] developed an integrated amperometry waveform for the direct detection of amino acids using high-performance anion-exchange chromatography (HPAEC) and integrated pulsed amperometric detection (IPAD). Subsequently, Jandik and co-workers [13-15] carried out some research on this method. This research included a new technique for increasing retention of arginine on an anion-exchange column, analyzing mixtures of amino acids and carbohydrates using bimodal integrated amperometric detection, and simplified in-line sample preparation for amino acid analysis in samples containing carbohydrate. However, the factors affecting the separation and detection of amino acids using HPAEC and IPAD have not been carefully studied. In this paper, some factors affecting the separation and detection of amino acids by HPAEC with IPAD were investigated. These factors include eluent concentration, column temperature, and detection waveform. The optimized gradient elution conditions and column temperature for analyzing 19 amino acids were obtained. The optimized method was applied to determine trace impurities of amino acid in a valine product.

\section{Experimental}

\subsection{Reagents}

Standard amino acids and sodium azide were purchased from Sigma-Aldrich (St Louis, MO, USA) and were used as received. For all purposes, water with a resistivity of $18 \mathrm{M} \Omega \mathrm{cm}$ was used. The single component stock solutions $(\sim 0.5 \mathrm{~m} M)$ of amino acids were prepared in water containing $20 \mathrm{mg} / 1$ sodium azide to prevent microbial growth. The injected standard mixtures of solutions of amino acids were made by diluting corresponding aliquots of the single component stock solutions of amino acids with an aqueous diluent containing $20 \mathrm{mg} / 1$ sodium azide.

Sodium hydroxide (Beijing Chemical Factory, China) and anhydrous sodium acetate (Dionex) were used to prepare the gradient mobile phases. The mobile phase of $250 \mathrm{~m} M$ sodium hydroxide was prepared by diluting an aliquot of $50 \%$ sodium hydroxide, which was prepared by dissolving solid sodium hydroxide in water. The resulting $50 \%$ solution was kept undisturbed for $24 \mathrm{~h}$ to allow the sodium carbonate to settle at the bottom prior to the last dilution step to $250 \mathrm{~m} M$. The $1 M$ sodium acetate mobile phase was prepared by dissolving an aliquot of anhydrous sodium acetate in water and filtering the solution through a $0.2-\mu \mathrm{m}$ nylon filter. All mobile phases were kept under nitrogen to prevent accumulation of atmospheric carbon dioxide.

\subsection{Instrumentation}

The chromatograph used for this work was a DX-600 IC system (Dionex, Sunnyvale, CA, USA). The system consisted of a GS50 gradient pump with on-line degassing, an AS50 autosampler, an AS50 thermal compartment, and an ED50 electrochemical detector equipped with a thin-layer type amperometric cell. The cell consisted of a gold electrode, a combination reference electrode of glass and $\mathrm{Ag} /$ $\mathrm{AgCl}(3 \mathrm{MCl})$ and titanium counter electrode consisting of the cell body. Separation was carried out using the AminoPac PA 10 column set consisting of a guard column (50 $\mathrm{mm} \times 2 \mathrm{~mm}$ I.D.) and an analytical column $(250 \mathrm{~mm} \times 2 \mathrm{~mm}$ I.D. $)$. The columns and the electrochemical detection cell were placed inside the AS50 thermal compartment for temperature control. The chromatographic system control, data acquisition and analysis were carried out using PeakNet 6.3 Software (Dionex). The sample injection volume was $25 \mu$ l. Gradient elution was carried out at a flow-rate of $0.25 \mathrm{ml} / \mathrm{min}$ with water, sodium hydroxide and sodium acetate mobile phases using the ternary gradient method of Table 1 . The integrated amperometry detection waveform is presented in Table 2. The temperature ranged between 25 and $40{ }^{\circ} \mathrm{C}$.

\section{Results and discussion}

\subsection{Effect of sodium hydroxide and sodium acetate eluent concentration on the separation of amino acids and the optimization of gradient elution conditions}

Sodium hydroxide and sodium acetate were selected as eluents for separating amino acids. The basic 
Table 1

Gradient conditions for analyzing 19 amino acids

\begin{tabular}{rllll}
\hline $\begin{array}{l}\text { Time } \\
(\mathrm{min})\end{array}$ & $\begin{array}{l}\mathrm{H}_{2} \mathrm{O} \\
(\%)\end{array}$ & $\begin{array}{l}250 \mathrm{~m} M \mathrm{NaOH} \\
(\%)\end{array}$ & $\begin{array}{l}1 M \mathrm{NaAc} \\
(\%)\end{array}$ & Curve $^{\mathrm{a}}$ \\
\hline 0.0 & 76 & 24 & & \\
2.0 & 76 & 24 & & 8 \\
8.0 & 64 & 36 & & 8 \\
11.0 & 64 & 36 & 40 & 8 \\
18.0 & 40 & 20 & 40 & 8 \\
21.0 & 40 & 20 & 60 & \\
23.0 & 20 & 20 & 60 & \\
35.0 & 20 & 20 & & \\
35.1 & 20 & 80 & & \\
37.1 & 20 & 80 & & \\
37.2 & 76 & 24 & & \\
60.0 & 76 & 24 & & \\
\hline
\end{tabular}

${ }^{\text {a }}$ Shapes of gradient curves are defined in the GS 50 Gradient Pump Operator's Manual, 37-38 (Dionex Document No. 031612, Revision 2). Curve 5 is linear while Curve 8 is one of the four available concave curves (6-9) with $20 \%$ change at about $60 \%$ of a time segment and $70 \%$ change at about $90 \%$ of the same programmed time segment.

solution of sodium hydroxide can make amino acids form anions. Therefore, amino acids can be separated on an anion-exchange column. Sodium hydroxide can elute weakly retained amino acids. Except for eluting amino acids, the strong basic medium of sodium hydroxide can give amperometric detector high sensitivity for the detection of amino acids. Sodium acetate was used to elute strongly retained amino acids.

We investigated the influence of sodium hydroxide concentration on the separation of amino acids. The

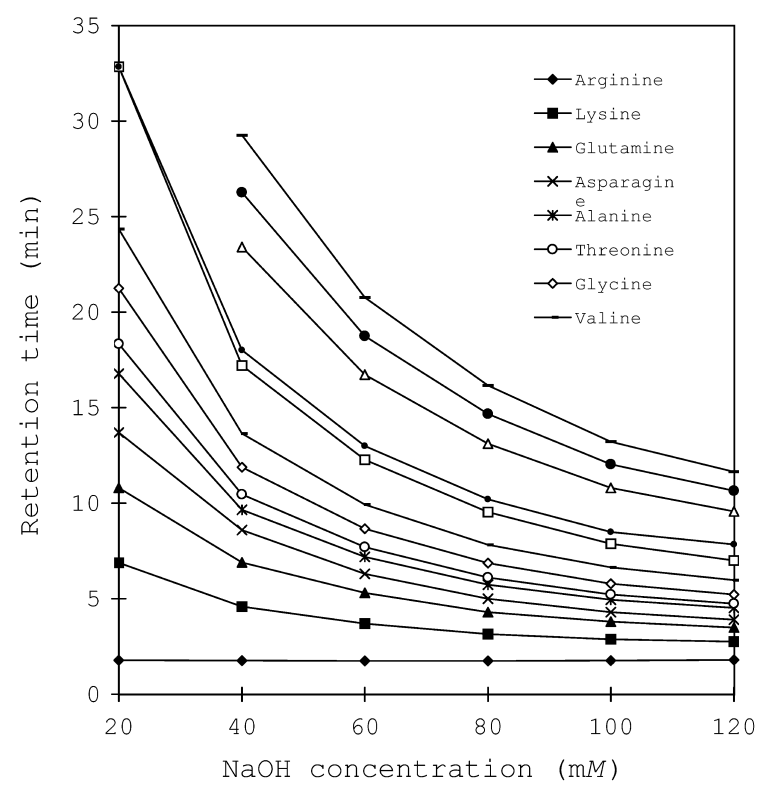

Fig. 1. Influence of sodium hydroxide eluent concentration on the retention time of weakly retained amino acids.

column temperature was maintained at $35{ }^{\circ} \mathrm{C}$ in the experiment. Fig. 1 illustrates the effect of sodium hydroxide concentration on the retention time of weakly retained amino acids. The retention times of these amino acids (except for arginine) decrease with increasing sodium hydroxide concentration. However, the elution order of amino acids is not changed. The results indicate that the selectivity changes in these weakly retained amino acids are slight with changing sodium hydroxide concentration.

Table 2

Detection waveforms for amino acids

\begin{tabular}{|c|c|c|c|c|c|}
\hline \multicolumn{3}{|c|}{ Waveform I } & \multicolumn{3}{|c|}{ Waveform II } \\
\hline $\begin{array}{l}\text { Time } \\
(\mathrm{ms})\end{array}$ & $\begin{array}{l}\text { Potential (V) versus } \\
\mathrm{Ag} / \mathrm{AgCl}(3 M \mathrm{KCl})\end{array}$ & $\begin{array}{l}\text { Current } \\
\text { integration }\end{array}$ & $\begin{array}{l}\text { Time } \\
(\mathrm{ms})\end{array}$ & $\begin{array}{l}\text { Potential (V) versus } \\
\mathrm{Ag} / \mathrm{AgCl}(3 M \mathrm{KCl})\end{array}$ & $\begin{array}{l}\text { Current } \\
\text { integration }\end{array}$ \\
\hline 0 & -0.20 & & 0 & -0.20 & \\
\hline 40 & -0.20 & & 40 & -0.20 & \\
\hline 50 & 0.00 & & 50 & 0.00 & \\
\hline 210 & 0.00 & Begin & 210 & 0.00 & Begin \\
\hline 220 & 0.27 & & 220 & 0.00 & \\
\hline 460 & 0.27 & & 460 & 0.00 & \\
\hline 470 & 0.00 & & 470 & 0.00 & \\
\hline 560 & 0.00 & End & 560 & 0.00 & End \\
\hline 570 & -2.00 & & 570 & -2.00 & \\
\hline 580 & -2.00 & & 580 & -2.00 & \\
\hline 590 & 0.60 & & 590 & 0.60 & \\
\hline 600 & -0.20 & & 600 & -0.20 & \\
\hline
\end{tabular}


If only sodium hydroxide solutions are used as eluent, strongly retained amino acids, such as glutamic acid, aspartic acid, cystine and tyrosine, are not eluted from the column even at an eluent concentration as high as $200 \mathrm{~m} M$ of sodium hydroxide. The strongly retained amino acids can be eluted only by a stronger eluent such as sodium acetate. The influence of sodium acetate concentration on the separation of the strongly retained amino acids was investigated under the conditions keeping the sodium hydroxide concentration at 80 $\mathrm{m} M$ and the column temperature at $35^{\circ} \mathrm{C}$. Fig. 2 shows the effect of sodium acetate concentration on the retention time of strongly retained amino acids. From Fig. 2, we can see that the selectivity variations between amino acids containing two carboxyl groups (glutamic acid and aspartic acid) and amino acids containing only one carboxyl group (phenylalanine) are different when the sodium acetate concentration is changed. The differences can be used to improve the separation between them. The reason for this phenomenon is that the amino acids consisting two carboxyl groups have higher charge than the amino acids consisting one carboxyl group. The difference in the retention time with changing eluent concentration is greater for high charge ions than for low charge ions in anion-exchange chromatography [16].

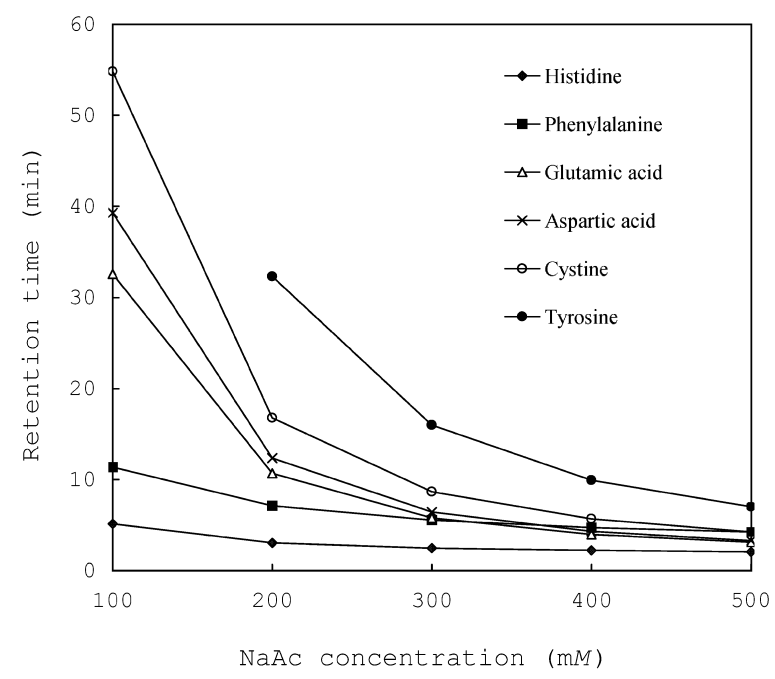

Fig. 2. Influence of sodium acetate eluent concentration on the retention time of strongly retained amino acids. Sodium hydroxide concentration is held constant at $80 \mathrm{mM}$.
The above results indicate that simultaneous separation of weakly and strongly retained amino acids in a single run requires gradient elution methods employing both sodium hydroxide and sodium acetate. An optimized gradient elution condition for the separation of 19 amino acids is given in Table 1. A chromatogram of 19 amino acids obtained under these conditions is shown in Fig. 3. From Fig. 3, we can see that 19 amino acids were completely separated.

\subsection{Influence of column temperature on the separation of amino acids}

The column temperature affects the retention of analytes in chromatographic analysis. We investigated the influence of column temperature on the separation of amino acids under the gradient condition of Table 1. The column temperatures were changed from 25 to $40{ }^{\circ} \mathrm{C}$. Fig. 4 shows the effect of column temperature on retention times of amino acids. From Fig. 4, we can see that the effect of temperature on the retention time of amino acids is complex. The retention times for valine, isoleucine and leucine are obviously increased with increasing temperature. However, the retention times for serine and methionine are obviously decreased with increasing temperature. For other amino acids, the retention time changes with varying column temperature are not obvious. The reason for the above phenomenon could be that the retention of amino acids in anion-exchange chromatography could be an endothermic process (retention to be increased with increasing temperature) or exothermic process (retention to be decreased with increasing temperature). Thus, significant but only slight selectivity changes in some weakly retained amino acids, which are eluted with sodium hydroxide eluent, can be achieved by changing the column temperature. The separation between two closely eluted amino acids can be improved by changing column temperature. For example, serine and proline make up the overlapping peak at $25^{\circ} \mathrm{C}$, but both are completely separated at $35^{\circ} \mathrm{C}$, with resolution of 1.84 . In another example, leucine and methionine form the overlapping peak at $40{ }^{\circ} \mathrm{C}$, but good separation is obtained between them at $35^{\circ} \mathrm{C}$, with resolution of 1.42. From the above results, the column temperature 


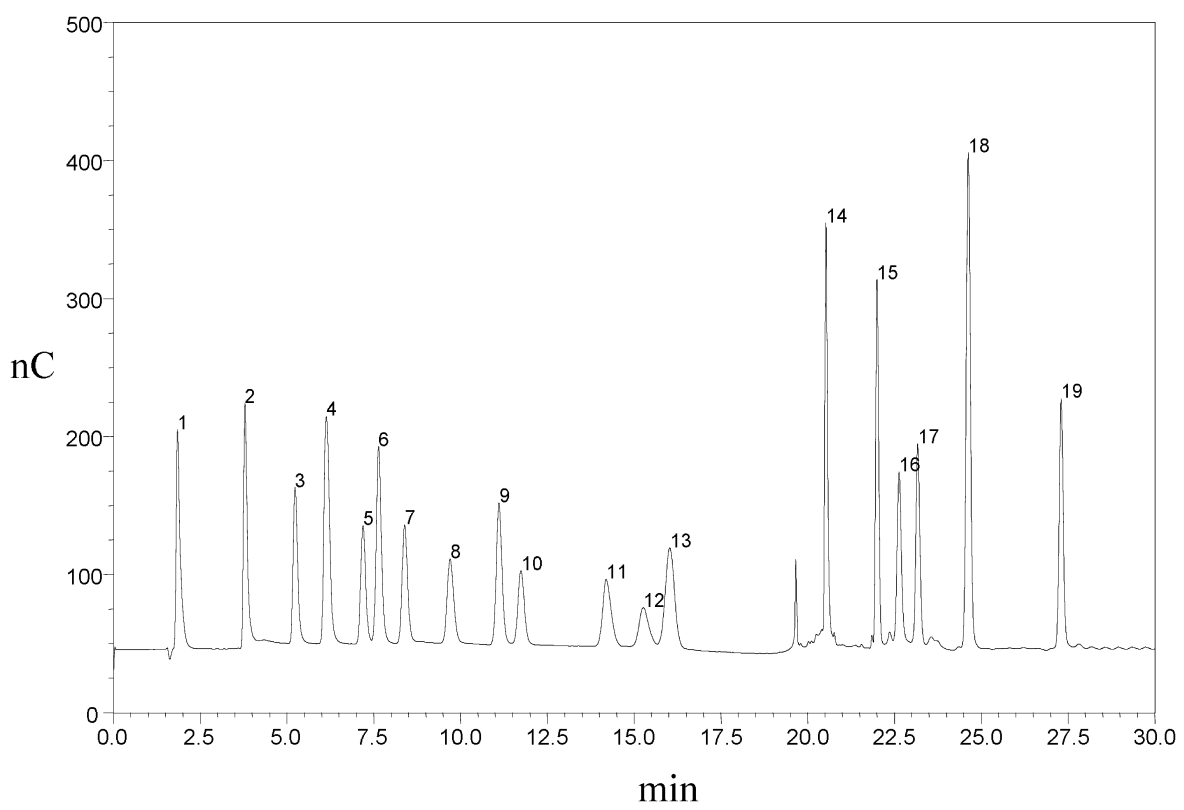

Fig. 3. Chromatogram of a standard solution of 19 amino acids. Chromatographic conditions: AminoPac PA 10 column set; gradient method from Table 1; detection waveform I of Table 2; column temperature $35^{\circ} \mathrm{C}$; flow-rate $0.25 \mathrm{ml} / \mathrm{min}$; injection volume $25 \mu \mathrm{l}$. Peak (concentration, $\mu M)$ : $1=$ arginine $(9.0) ; 2=$ lysine $(24.0) ; 3=$ glutamine $(11.6) ; 4=$ asparagine $(13.2) ; 5=$ alanine $(12.2) ; 6=$ threonine $(9.3)$; $7=$ glycine $(12.0) ; 8=$ valine $(15.1) ; 9=$ serine $(8.3) ; 10=$ proline $(12.2) ; 11=$ isoleucine $(26.5) ; 12=$ leucine $(24.6) ; 13=$ methionine $(12.8)$; $14=$ histidine $(7.5) ; 15=$ phenylalanine $(11.6) ; 16=$ glutamic acid (26.7); $17=$ aspartic acid $(19.2) ; 18=$ cystine $(7.5) ; 19=$ tyrosine $(8.2)$.

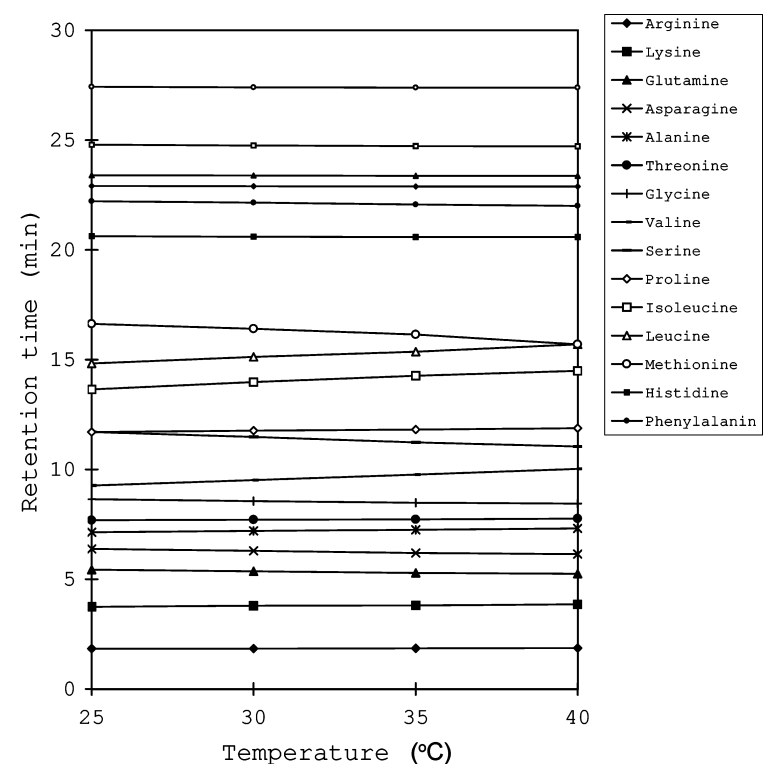

Fig. 4. Effect of column temperature on the retention times of amino acids. of $35^{\circ} \mathrm{C}$ is suitable for the separation of 19 amino acids.

\subsection{Effect of sodium hydroxide and sodium acetate eluent concentration on the detection of amino acids}

Amino acids in strongly alkaline solution can be detected with IPAD [12,14,17,18]. We investigated the effects of sodium hydroxide and sodium acetate eluent concentration on the detection of amino acids. The waveform I of Table 2 is employed in this experiment. Fig. 5 shows the effect of sodium hydroxide concentration on the detection of weakly retained amino acids. From Fig. 5, we can see that the peak areas of amino acids increase with increasing sodium hydroxide concentration in the range of lower concentration, but they are nearly invariant or slightly decreased with increasing sodium hydroxide concentration in the range of higher concentration. The reasons for the phenomenon are probably related to the response of the gold electrode and the change 


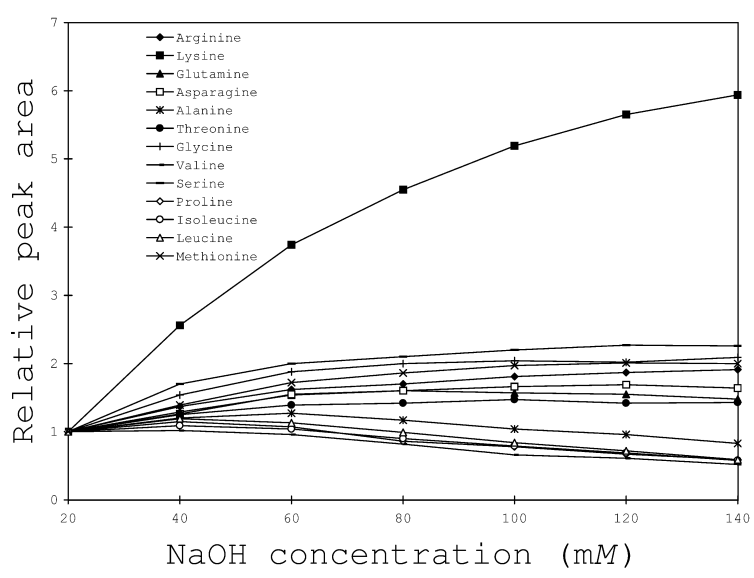

Fig. 5. Effect of sodium hydroxide eluent concentration on the detection of weakly retained amino acids.

in the dissociation equilibriums of amino acids under the different basic conditions. The above results indicate that the detection sensitivity of amino acids can be improved by increasing the concentration of sodium hydroxide in a certain concentration range that is different for each amino acid (Fig. 5), especially for lysine, serine, glycine and methionine. The detection limit of lysine obtained with $140 \mathrm{mM}$ $\mathrm{NaOH}$ eluent $(0.15 \mathrm{pmol})$ is about eight times as low as that obtained with $20 \mathrm{mM} \mathrm{NaOH}$ eluent $(1.22$ pmol). Under the elution conditions for the optimum separation of amino acids, detection sensitivity of amino acids can be improved by means of postcolumn addition of sodium hydroxide.

Sodium acetate concentration affects the detection of strongly retained amino acids. Fig. 6 illustrates the effect of sodium acetate concentration on the detection of strongly retained amino acids. The peak areas of amino acids increase with the increase in sodium acetate concentration in the range of lower concentration, but the peak areas of amino acids are nearly invariant or slightly decreased with increasing sodium acetate concentration in the range of higher concentration. The above results indicate that the detection sensitivity of strongly retained amino acids can be improved by increasing the concentration of sodium acetate in a certain concentration range (Fig. 6), especially for glutamic acid, aspartic acid, cystine and tyrosine. The detection limit of aspartic acid obtained with $500 \mathrm{mM}$ NaAc eluent $(0.40 \mathrm{pmol})$ is

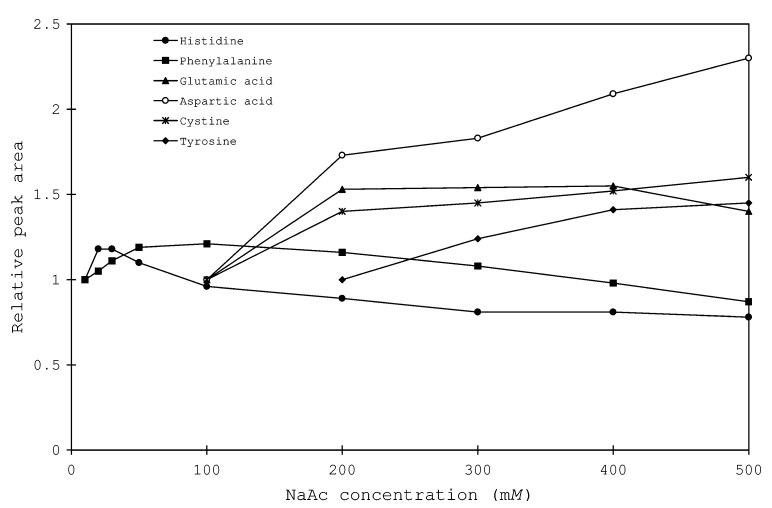

Fig. 6. Effect of sodium acetate eluent concentration on the detection of strongly retained amino acids. Sodium hydroxide concentration is held constant at $80 \mathrm{mM}$.

about 10 times as low as that obtained with $100 \mathrm{mM}$ $\mathrm{NaAc}$ eluent (4.08 pmol).

\subsection{Detection of amino acids at different waveforms}

The waveform I in Table 2 is a basic IPAD waveform for detection of amino acids. We investigated the response of amino acids at different sampling step potentials (the potentials between 220 and $460 \mathrm{~ms}$ ) that are $0.27,0.22,0.17,0.12,0.07$, 0.02 and $0.00 \mathrm{~V}$. The peak areas of the amino acids that do not contain a hydroxyl group (non-hydroxyl amino acids) show a marked decrease with decreasing sampling step potential from 0.27 to $0.00 \mathrm{~V}$. On the other hand, the peak areas of the amino acids containing hydroxyl groups (hydroxyl amino acids) decrease only slightly with the decrease in the sampling step potential from 0.27 to $0.00 \mathrm{~V}$. Thus, if the sampling step potentials in waveform I are changed from 0.27 to $0.00 \mathrm{~V}$, a modified IPAD waveform (waveform II in Table 2) is obtained. The hydroxyl amino acids can be detected with waveform II, but the non-hydroxyl amino acids are not detected or the detection responses are very weak. This indicates that the detection potential of non-hydroxyl amino acids is higher than that of hydroxyl amino acids. The reason is that the electrochemical detection mechanisms for aliphatic hydroxyl groups and for amino acids not containing hydroxyl groups are different [18]. Therefore, hydroxyl amino acids 


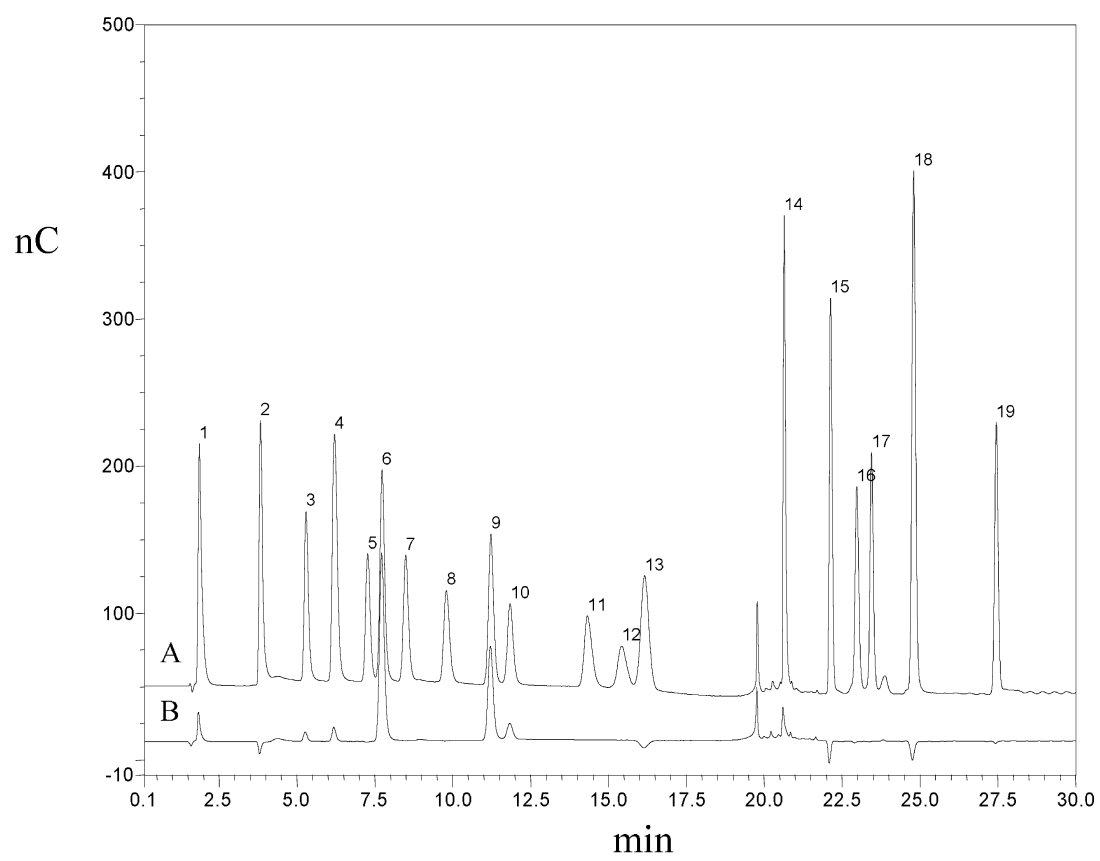

Fig. 7. Comparison of two different detection waveforms for the detection of amino acids. Chromatogram A: detection waveform I of Table 2; chromatogram B: detection waveform II of Table 2. Other chromatographic conditions, peak identities and the analyte amounts are given in Fig. 3.

can be selectively detected by choosing a modified detection waveform. Fig. 7 shows the chromatograms obtained with two different waveforms. From Fig. 7, we can see that the peaks of all amino acids were observed using detection waveform I. However, when detection waveform II was employed, the peaks of hydroxyl amino acids were still observed, but the peaks of non-hydroxyl amino acids either disappeared or were much reduced in size. The method employing the two different detection waveforms can thus be useful for identifying hydroxyl amino acids and non-hydroxyl amino acids in an unknown sample.

\subsection{Analytical method performance parameters}

Analytical performance parameters were measured under gradient conditions of Table 1, detection waveform I of Table 2, column temperature of $35^{\circ} \mathrm{C}$ and injection volume of $25 \mu$. These parameters are given in Table 3. The detection limits were calculated as three times the signal (peak height)-to-noise ratios. The RSD values of peak areas were determined by making five repetitive injections of amino acids. The calibration graphs using peak areas were linear for all the analytes about three orders of magnitude.

\subsection{Analysis of samples}

The above optimized conditions for determination of 19 amino acids was obtained through investigating the factors affecting separation and detection of amino acids. The method was applied to determine trace amino acid impurities in valine product. Valine product is a kind of amino acid product. The determination of amino acid impurities in valine product must be carried out when identifying the quality of the product. An accurately weighed valine solid sample $(\sim 15 \mathrm{mg})$ was dissolved in $10 \mathrm{ml}$ $20 \mathrm{mg} / \mathrm{ml}$ sodium azide solution and diluted with $20 \mathrm{mg} / \mathrm{l}$ sodium azide solution to $100 \mathrm{ml}$ to prepare the sample solution. All sample solutions were filtered through a $0.45-\mu \mathrm{m}$ membrane filter prior to 
Table 3

Summary of method performance parameters

\begin{tabular}{|c|c|c|c|c|c|}
\hline Analyte & $\begin{array}{l}\text { Detection limit } \\
(\mathrm{pmol}) \\
(S / N=3)\end{array}$ & $\begin{array}{l}\text { Linear } \\
\text { range } \\
(\mu M)\end{array}$ & $\begin{array}{l}\text { Regression equation } \\
y=\text { peak area }(\mathrm{nC} \min ) \\
x=\text { concentration }(\mu M)\end{array}$ & $\begin{array}{l}\text { Correlation } \\
\text { coefficient } \\
(n=5)\end{array}$ & $\begin{array}{l}\text { RSD } \\
(\%) \\
(n=5)\end{array}$ \\
\hline Arginine & 0.36 & $0.05-20$ & $y=1.53 x+3.96$ & 0.9973 & 1.5 \\
\hline Lysine & 0.77 & $0.10-60$ & $y=0.59 x+7.87$ & 0.9942 & 1.0 \\
\hline Glutamine & 0.54 & $0.05-20$ & $y=1.17 x+2.74$ & 0.9999 & 1.6 \\
\hline Asparagine & 0.51 & $0.05-20$ & $y=1.50 x+6.07$ & 0.9998 & 2.0 \\
\hline Alanine & 0.80 & $0.10-30$ & $y=0.84 x+2.10$ & 0.9984 & 2.7 \\
\hline Threonine & 0.32 & $0.05-40$ & $y=2.57 x-0.06$ & 0.9999 & 2.2 \\
\hline Glycine & 0.74 & $0.10-30$ & $y=1.08 x+2.22$ & 0.9990 & 1.3 \\
\hline Valine & 1.31 & $0.10-40$ & $y=0.75 x+1.18$ & 0.9996 & 1.6 \\
\hline Serine & 0.43 & $0.10-20$ & $y=2.72 x+0.73$ & 0.9999 & 0.6 \\
\hline Proline & 1.28 & $0.10-30$ & $y=1.29 x+0.07$ & 0.9995 & 1.5 \\
\hline Isoleucine & 3.04 & $0.20-80$ & $y=0.70 x+0.73$ & 0.9999 & 2.8 \\
\hline Leucine & 4.52 & $0.20-60$ & $y=0.66 x+0.05$ & 0.9997 & 3.6 \\
\hline Methionine & 1.02 & $0.10-40$ & $y=2.03 x+2.37$ & 0.9978 & 1.9 \\
\hline Histidine & 0.15 & $0.05-10$ & $y=2.05 x+9.26$ & 0.9873 & 5.6 \\
\hline Phenylalanine & 0.26 & $0.05-30$ & $y=1.70 x+5.58$ & 0.9966 & 1.6 \\
\hline Glutamic acid & 1.25 & $0.10-80$ & $y=0.37 x+3.94$ & 0.9881 & 3.7 \\
\hline Aspartic acid & 0.74 & $0.10-50$ & $y=0.62 x+3.86$ & 0.9961 & 3.5 \\
\hline Cystine & 0.24 & $0.05-20$ & $y=4.85 x+2.56$ & 0.9812 & 1.7 \\
\hline Tyrosine & 0.48 & $0.05-20$ & $y=2.36 x+2.36$ & 0.9994 & 1.5 \\
\hline
\end{tabular}

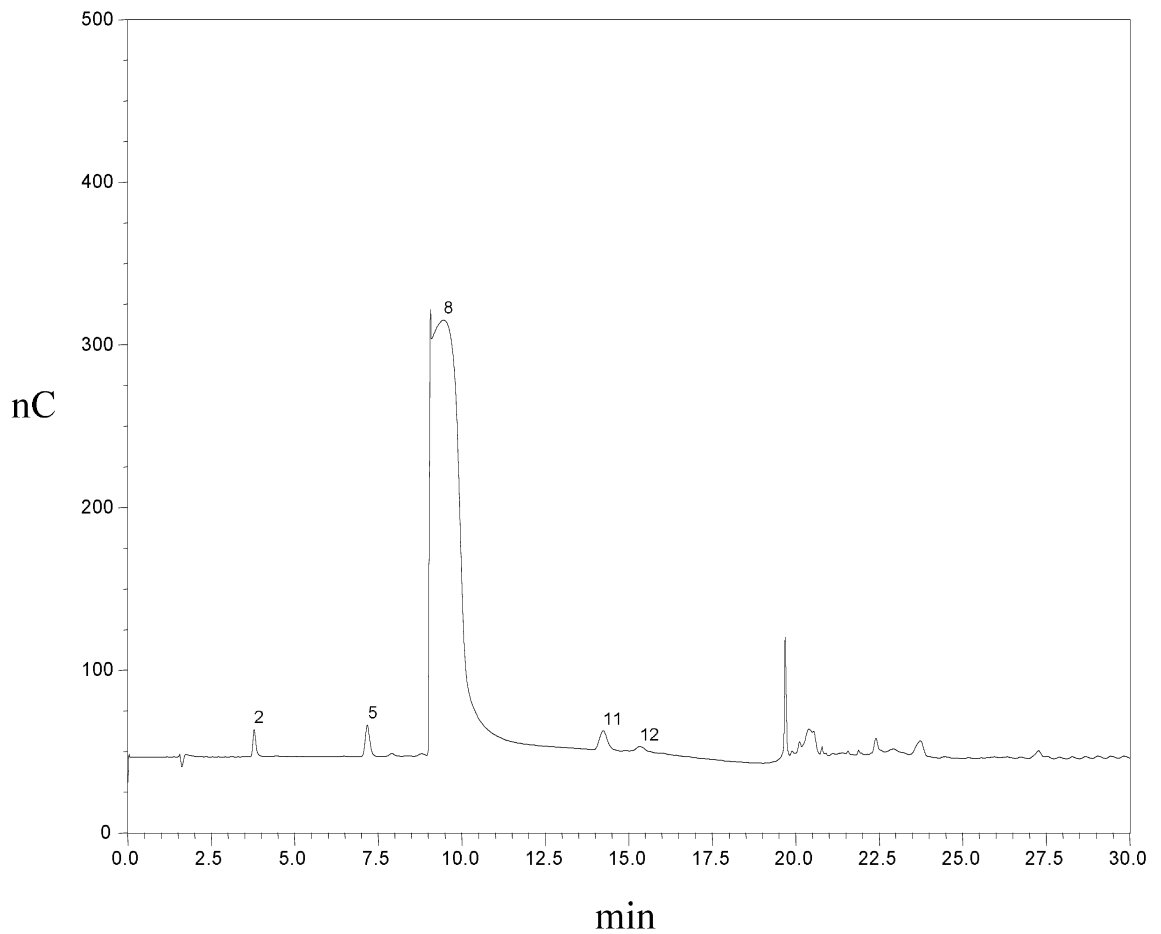

Fig. 8. Chromatogram of a sample solution of valine product. The chromatographic conditions and peak identities are given in Fig. 3. 
Table 4

The results of analysis and recovery for valine product

\begin{tabular}{lllll}
\hline Component & $\begin{array}{l}\text { Detected level in } \\
\text { sample }(\mathrm{mg} / \mathrm{g})\end{array}$ & $\begin{array}{l}\text { RSD } \\
(\%)\end{array}$ & $\begin{array}{l}\text { Recovery } \\
(\%)\end{array}$ & $\begin{array}{l}\text { RSD } \\
(\%)\end{array}$ \\
\hline Lysine & 1.04 & 2.2 & 94.2 & 3.0 \\
Alanine & 0.95 & 2.5 & 92.6 & 3.1 \\
Isoleucine & 2.62 & 4.5 & 96.4 & 4.7 \\
Leucine & 0.65 & 5.1 & 90.3 & 5.4 \\
\hline
\end{tabular}

All data are the average values for five determinations.

injection. The chromatogram of a sample solution of valine product is shown in Fig. 8. The large amount of valine does not interfere with the determination of trace amino acid impurities. We identified four kinds of amino acid impurities in the valine product by spiking with known standards. The peak area calibration graphs were used for quantitative determination. Recovery experiments were carried out by adding known amounts of amino acids to the sample solution of valine product under the same conditions as for analyzing the samples. Table 4 summarizes the analytical results and recoveries of identified components in the analyzed samples. The results demonstrate both high accuracy and good precision of the method.

\section{Conclusions}

Eluent concentration, column temperature and detection waveform affect the separation and detection of amino acids by HPAEC and IPAD. When sodium hydroxide eluent concentration is changed, the selectivity changes in weakly retained amino acids are slight. When sodium acetate eluent concentration is changed, the selectivity variations between strongly retained amino acids containing two carboxyl groups and containing only one carboxyl group are obviously different. Significant but slight selectivity changes in some amino acids can be achieved by means of changing the column temperature. Detection sensitivity of amino acids can be improved by increasing the concentration of sodium hydroxide and sodium acetate in a certain concentration range. The amino acids containing hydroxyl groups can be selectively detected by choosing a modified detection waveform. The optimized gradient elution conditions and column temperature for separating 19 amino acids were obtained. As an application example, we analysed trace amino acid impurities in valine product.

\section{References}

[1] D.H. Spackman, W.H. Stein, S. Moore, Anal. Chem. 30 (1958) 1190

[2] K.A. Piez, L. Morris, Anal. Biochem. 1 (1960) 187.

[3] P.B. Hamilton, Anal. Chem. 35 (1963) 2055.

[4] A.P. Williams, J. Chromatogr. 373 (1986) 175.

[5] R.S. Ersser, J.F. Davey, Med. Lab. Sci. 48 (1991) 59.

[6] D. Fekkes, J. Chromatogr. B 682 (1996) 3.

[7] P. Fürst, L. Pollack, T.A. Graser, H. Godel, P. Stehle, J. Chromatogr. 499 (1990) 557.

[8] K.L. Woo, Amino acid analysis protocols, in: C. Cooper, N. Packer, K. Williams (Eds.), Methods in Molecular Biology, Vol. 159, Humana Press, Totowa, NJ, 2001, p. 141.

[9] K.R. Kim, J.H. Kim, E.J. Cheong, C.M. Jeong, J. Chromatogr. A 722 (1996) 303.

[10] J.T. Smith, Electrophoresis 18 (1997) 2377.

[11] J.T. Smith, Electrophoresis 20 (1999) 3078.

[12] A.P. Clarke, P. Jandik, R.D. Rocklin, Y. Liu, N. Avdalovic, Anal. Chem. 71 (1999) 2774.

[13] P. Jandik, J. Cheng, D. Jensen, S. Manz, N. Avdalovic, Anal. Biochem. 287 (2000) 38.

[14] P. Jandik, A.P. Clarke, N. Avdalovic, D.C. Andersen, J. Cacia, J. Chromatogr. B 732 (1999) 193.

[15] P. Jandik, J. Cheng, D. Jensen, S. Manz, N. Avdalovic, J. Chromatogr. B 758 (2001) 189.

[16] D.T. Gjerde, G. Schmuckler, J.S. Fritz, J. Chromatogr. 187 (1980) 35.

[17] L.E. Welch, W.R. LaCourse, D.A. Mead Jr, D.C. Johnson, T. Hu, Anal. Chem. 61 (1989) 555.

[18] D.C. Johnson, W.R. LaCourse, Anal. Chem. 62 (1990) 589A. 